\title{
Expression of immune checkpoint PD-1 in non-small cell lung cancer is associated with tumor cell DNA-dependent protein kinase
}

\author{
MARIKA SAAR $^{1-3}$, JAANIKA NARITS $^{3}$, LAURA MÄGI $^{3}$, HARDI AASPÕLLU $^{3}$, ANNETT VAPPER $^{3}$, \\ MARJU KASE ${ }^{3,4}$, AVE MINAJEVA $^{5}$, TÕNU VOODER ${ }^{6}$, HANNES TAMM $^{5,7}$, \\ MAKSIM BULDAKOV $^{5,7}$, DARJA LAVÕGINA ${ }^{3}$ and JANA JAAL ${ }^{3,4}$ \\ ${ }^{1}$ Department of Pharmacy, Tartu University Hospital; ${ }^{2}$ Pharmacy Institute, University of Tartu; ${ }^{3}$ Institute of Clinical Medicine, \\ Faculty of Medicine, University of Tartu; ${ }^{4}$ Department of Radiotherapy and Oncological Therapy, Haematology and \\ Oncology Clinic Tartu University Hospital, Tartu 50406; ${ }^{5}$ Institute of Biomedicine and Translational Medicine, \\ Faculty of Medicine, University of Tartu, Tartu 50411, Estonia; ${ }^{6}$ Helios Clinics, Center for Thoracic and Lung Surgery, \\ D-47805 Krefeld, Germany; ${ }^{7}$ Pathology Department, Tartu University Hospital, Tartu 50406, Estonia
}

Received January 6, 2021; Accepted June 28, 2021

DOI: $10.3892 / \mathrm{mco} .2021 .2369$

\begin{abstract}
Immunotherapy using immune checkpoint inhibitors has demonstrated durable responses and has significantly improved survival in patients with non-small cell lung cancer (NSCLC). Moreover, immunotherapy is increasingly used in combination with cytotoxic treatments such as chemotherapy and radiotherapy. Although the combined treatments are more effective, the underling mechanisms that lead to higher antitumor activity are not fully understood. Therefore, the aim of the current retrospective study was to determine the relationship between expression of immune checkpoints [programmed cell death protein 1 (PD-1) and programmed death-ligand 1 (PD-L1)] and the enzyme DNA-dependent protein kinase (DNA-PK), which is part of a key pathway involved in the repair of cytotoxic cancer therapy induced damage. Surgically excised NSCLC tissues $(n=121)$ were histologically examined for overall extent of inflammation (score 0-3). Expression levels of PD-1 (number of PD-1 positive cells), PD-L1 [tumor proportion score (TPS); \%] and DNA-PK (proportion of DNA-PK positive tumor cells; \%) were determined using immunohistochemistry. Expressions of these markers were compared in different clinicopathological subgroups and later used for nonparametric Spearman correlation analysis to determine associations. In patients with NSCLC, PD-1 was significantly expressed in males $(\mathrm{P}=0.030)$ and in patients with no or trivial inflammation scores $(\mathrm{P}=0.030)$. $\mathrm{PD}-\mathrm{L} 1$ expression was also
\end{abstract}

Correspondence to: Mrs. Marika Saar, Department of Pharmacy, Tartu University Hospital, Puusepa 8, Tartu 50406, Estonia E-mail: marika.Saar@kliinikum.ee

Key words: non-small cell lung cancer, immune checkpoints, programmed cell death protein 1 , inflammation, DNA-dependent protein kinase significantly higher in current smokers $(\mathrm{P}=0.025)$. Correlation analysis was based on the individual values of patients and revealed a significant association between one of the targets of immune checkpoint inhibitors and tumor cell DNA-PK. Tumors with higher numbers of PD-1 positive cells also demonstrated higher tumor cell DNA-PK expressions $(\mathrm{P}=0.027)$. The results demonstrated a significant positive correlation between the PD-1/PD-L1 axis and tumor cell DNA-PK expression in patients with NSCLC. Further studies are required to clarify the significance of this correlation and its effect on the efficacy of immunotherapy and cytotoxic cancer therapy combinations.

\section{Introduction}

Lung cancer is a leading cause of cancer-related deaths worldwide and number of lung cancer deaths is estimated to increase by $86 \%$ by $2035(1,2)$.

For years, standard treatments for the patients with advanced metastatic non-small cell lung cancer (NSCLC) included cytotoxic chemotherapy and specific inhibitors for NSCLC subtypes, harboring epidermal growth factor receptor (EGFR) mutations or chromosomal rearrangements of anaplastic lymphoma kinase (ALK) (3). As a result, the patient with advanced NSCLC had a median survival of approximately 1 year after chemotherapy and 2-3 years after the treatment with EGFR tyrosine kinase inhibitors $(4,5)$.

Recently, immunotherapy with immune checkpoint inhibitors has demonstrated durable responses and significantly improved survival rate in the patients with NSCLC. Programmed death protein 1 (PD-1) and its ligand (PD-L1) are the first introduced checkpoints being targeted in NSCLC and according to several clinical trials, antibodies against PD-1 and PD-L1 have significant efficacy in both, the first line and the second line treatment of metastatic NSCLC (6-9). Despite of demonstrated successes, response to the immunotherapy interventions is seen only in a subset $(\leq 5 \%)$ of patients $(10)$. Therefore, a substantial amount of current research is focused 
on improved performance of immunotherapies with novel combinations (including cytotoxic agents) together with biomarker optimization $(11,12)$. In fact, the immunotherapy and chemotherapy combinations that are already proven to be more effective are now entered into clinical practice of metastatic NSCLC management $(9,13)$.

To date, the most advanced biomarker for NSCLC is an immunohistochemical expression level of PD-L1 on the tumor cells. In different NSCLC cell line models and between patients, the expression levels of PD-L1 may vary significantly (14), whereas several phase-III clinical studies have reported better treatment responses in patients with higher tumor proportion score of PD-L1 (7-9). Previous reports additionally showed that the treatment efficacy of immune checkpoint inhibitors also correlated with the molecular smoking signature, higher neoantigen burden, and DNA repair pathway mutations that all lead to high mutational burden $(15,16)$. Furthermore, it has been reported that pathogenic DNA damage response (DDR) and repair mutations are associated with improved treatment response rate, progression-free survival, and overall survival in patients with NSCLC treated with programmed death ligand 1 [PD-(L)1] inhibitor therapy (17). Therefore, it is suggested that combining DDR inhibitors with DNA damaging agents, or PD-1 blockade can enhance the overall DNA damage and induce a more sustained upregulation of the cGAS-STING pathway and consequently the production of Th1 cytokines (18).

Changes in DNA repair machinery are especially important, when combinations of immune checkpoint inhibitors and conventional cytotoxic anti-cancer therapies (chemotherapy, radiotherapy) are in consideration. It has been demonstrated that an ability of cancer cells to repair therapeutically induced DNA damage has extensive impacts on anti-cancer therapeutic efficacy (19). Moreover, since DDR inhibition can also induce and amplify DNA damage in cancer cells, combining DDR inhibitors with immune checkpoint inhibitors and/or cytotoxic therapies represents an attractive strategy to improve patient outcomes (18).

DNA-dependent protein kinase catalytic subunit (DNA-PKcs), encoded by PRKDC/XRCC7 gene, is a pivotal DNA damage response (DDR) player (20). Along with $\mathrm{Ku}$ heterodimer, DNA-PKcs forms the DNA-PK holoenzyme complex. Of note, Ku heterodimer consists of Ku70 (XRCC6) and Ku80 (XRCC5) subunits. DNA-PK holoenzyme complex is a core component of non-homologous end joining (NHEJ) machinery, which alongside homologous recombination (HR), comprise the two major canonical pathways for DNA double strand breaks (DSBs) repair $(21,22)$. Expression and functioning of DNA-PK in several NSCLC cell lines has been reported (23), whereas the inhibition of DNA-PK was shown to enhance chemosensitivity as well as radiosensitivity in these cells $(24,25)$. Despite of that, interactions between PD-1/PD-L1 axis and DNA-PK are not fully explored.

The aim of our retrospective study was to describe the relationship between the expression of immune checkpoints PD-1 and PD-L1 and an enzyme DNA-PK, which is a part of key pathway for a repair of cancer therapy induced damage. For that, we carried out the immunohistochemical staining of the proteins of interest in the biopsy specimen obtained from patients thus providing a unique insight into the tumor and its microenvironment which includes the components of immune system.

\section{Materials and methods}

Patients and tissue samples. Tumor tissue samples were obtained from 121 patients with NSCLC, who were operated at Tartu University Hospital. None of patients received any cytotoxic drugs before surgery. Clinicopathological features of the recruited patients including age, sex, histologic type, and smoking status were collected from their medical records. The study was approved by the Reseach Ethics Committee of University of Tartu.

Microscopy and immunohistochemistry (IHC). Surgically excised NSCLC specimens were immediately fixed in buffered $10 \%$ formaline ( $\mathrm{pH} 7.4$ ) for $24 \mathrm{~h}$ and was subsequently embedded into paraffin wax (as routinely performed). Using the embedded tissue blocks, serial paraffin sections of $4 \mu \mathrm{m}$ were cut and were placed on glass slides for standard IHC.

Haematoxylin and eosin-stained sections were used for primary diagnosis of NSCLC. The diagnosis was confirmed by two independent pathologists. Afterwards, overall extent of inflammation was estimated in the tumor tissue. This was based on typical visual appearance of inflammation, including presence of edema and inflammatory cellular infiltration. For this analysis, an arbitrary score ranging from 0 to 3 ( 0 , no inflammation; 1 , weak; 2 , moderate; 3 , strong inflammatory reaction) was used.

For immunostaining, solutions and buffers purchased from Dako $\mathrm{GmbH}$ were used. The sections were deparaffinized and were incubated in target retrieval solution ( $\mathrm{pH} 9.0$ ) in $96^{\circ} \mathrm{C}$ thermostated water bath for $40 \mathrm{~min}$ and afterwards in peroxidase blocking solution for $5 \mathrm{~min}$ at room temperature. Subsequently, the tissue sections were incubated with specific anti-human PD-1 (1:100; Thermo Fisher Scientific, Inc., \#PA5-32543), PD-L1 (1:50; Thermo Fisher Scientific, Inc., \#PA5-20343) or DNA-PK (1:100; Santa Cruz Biotechnology; \#sc-390849) antibody at room temperature for $1 \mathrm{~h}$ under humid conditions. After several washings, the antigen-antibody complex was visualized by using Dako REAL ${ }^{\mathrm{TM}}$ EnVision Detection System, Peroxidase/DAB+, Rabbit/Mouse. The slides were counterstained with hematoxyline, dehydrated and cover-slipped for light microscopy.

Since a number of studies showed significant discrepancies in PD-L1 detection using different antibodies (26), we decided to perform additional immunostaining with clinically validated PD-L1 antibody. For this, we used PD-L1 ICH 22C3 pharmDx (Dako; Agilent Technologies, Inc.) kit which is a qualitative immunohistochemical assay with monoclonal mouse anti-PD-L1; a clone 22C3 intended for use in the detection of PD-L1 in formalin-fixed, paraffin-embedded NSCLC tissue. This particular staining was performed in accredited hospital laboratory using EnVision FLEX visualization system on Autostainer Link 48 according to the PD-L1 ICH 22C3 pharmDx (Dako; Agilent Technologies, Inc.) protocol.

Staining interpretation of PD-1, PD-L1 (PA5-20343, 22C3 pharmDx) and DNA-PK expression. Evaluation of IHC stained slides was carried out in a blinded fashion by the two authors independently in 10 randomly taken high power microscopic fields (magnification, x400).

At first, immunohistochemical expression of PD-1 was determined as a number of PD-1 positive cells per 
Table I. Characteristics of patients with non-small cell lung cancer $(n=121)$.

\begin{tabular}{lcc}
\hline Variable & No of patients $(\mathrm{n}=121)$ & Percentage $(\%)$ \\
\hline Sex & & 79 \\
Male & 32 & 26 \\
Female & $67(36-81)$ & - \\
Mean age, years (range) ${ }^{\mathrm{a}}$ & & 46 \\
Histology & 56 & 54 \\
Adenocarcinoma & 65 \\
Squamous cell cancer & & 79 \\
Smoking status & 95 & 14 \\
Current smoker & 17 \\
Non-smoker & 9 \\
Unknown & & 7 \\
\hline
\end{tabular}

${ }^{\mathrm{a}} \mathrm{Age}$ at the time of surgery.

microscopic field. Expression of PD-L1 (PA5-20343) on tumor cells was evaluated using an arbitrary score $(0$, no staining; 1, weak staining; 2, moderate staining; 3, strong staining). Proportion of DNA-PK positive tumor cells (\%) was calculated per microscopic field.

An experienced and qualified pathologist performed the evaluation of clinically relevant PD-L1 antibody. The expression of PD-L1 (22C3 pharmDx) was determined by using tumor proportion score (TPS, \%), which is a percentage of viable tumor cells showing partial or complete membrane staining at any intensity.

Statistical analysis. SPSS statistical software was used to calculate individual means, group means, and standard deviations of the mean. To determine statistical significance between the two data sets, we used Student's t-test. For all the selected parameters (PD-1, PD-L1, DNA-PK), we compared the means of following groups: Male vs. female, adenocarcinoma vs. squamous cell cancer, current smoker vs. non-smoker, no or trivial inflammation (score 0-2) vs. strong inflammation (score 3). Additionally, a nonparametric Spearman correlation analysis was utilized to check an association between PD-1/PD-L1 and DNA-PK. P-value $<0.05$ was considered as statistically significant.

Analysis of publicly available information regarding DNA-PK, PD-L1 and PD-1 mRNA and protein expression in lung cancer tissue. For comparison of our IHC data obtained from patient biopsy specimen to the publicly available mRNA sequencing data from The Cancer Genome Atlas (TCGA) database, we carried out correlation analysis on the following webpage: http:/gepia.cancer-pku.cn/detail.php?clicktag=correlation (27). The chosen parameters were as follows: gene names - PRKDC for DNA-PK, CD-274 for PD-L1, PDCD1 for PD-1; cancer name - LUAD for lung adenocarcinoma, LUSC for lung squamous carcinoma; correlation coefficient: Spearman. The results are presented in the Figs. S1 and S2. The information regarding the immunostaining of proteins of interest in the lung cancer-derived tissue slices was retrieved from the Human
Protein Atlas (HPA) database: for DNA-PK, https://www. proteinatlas.org/ENSG00000253729-PRKDC/pathology/

lung+cancer\#img; for PD-L1, https:/www.proteinatlas.org/ ENSG00000120217-CD274/pathology/lung+cancer\#img; for PD-1, https://www.proteinatlas.org/ENSG00000188389PDCD1/pathology/lung+cancer\#img.

\section{Results}

The clinicopathological characteristics of the recruited patients are summarized in Table I. Among 121 patients, 89 patients were males (74\%) and 32 were females (26\%), with a mean age of 67 years (range, 36-81 years). Fifty-six patients (46\%) had adenocarcinoma and 65 patients $(54 \%)$ were diagnosed of squamous cell cancer. Majority of the patients (79\%) were current smokers.

Extent of inflammation in NSCLC tumor tissues. Overall extent of inflammation estimated in the tumor tissue was based on a typical visual appearance of inflammation, including presence of edema and inflammatory cellular infiltration (score 0-3). Fig. 1 illustrates NSCLC tissue with trivial (Fig. 1A) and strong (Fig. 1B) inflammation features. Predominantly, the tissue sections showed moderate inflammatory features, which was followed by strong and trivial or no inflammation (moderate: 36\%, strong: 30\%, trivial: 29\% and no inflammation: $5 \%$, respectively).

Expression of PD-1, PD-L1 and DNA-PK in NSCLC tissues. Almost all immunohistochemical parameters were examined by two independent researchers and showed good accordance to each other $(\mathrm{P}<0.0005)$. Experienced and qualified pathologist performed the evaluation of clinically relevant PD-L1 antibody (22C3 pharmDx) staining.

Fig. 1 represents NSCLC tissues with a few (Fig. 1C) and numerous (Fig. 1D) PD-1 positive cells, tumors with negative (Fig. 1E) and positive PD-L1 (Fig. 1F) expression (22C3 pharmDx) as well as NSCLC samples with trivial (Fig. 1G) and noteworthy (Fig. 1H) DNA-PK expression. 

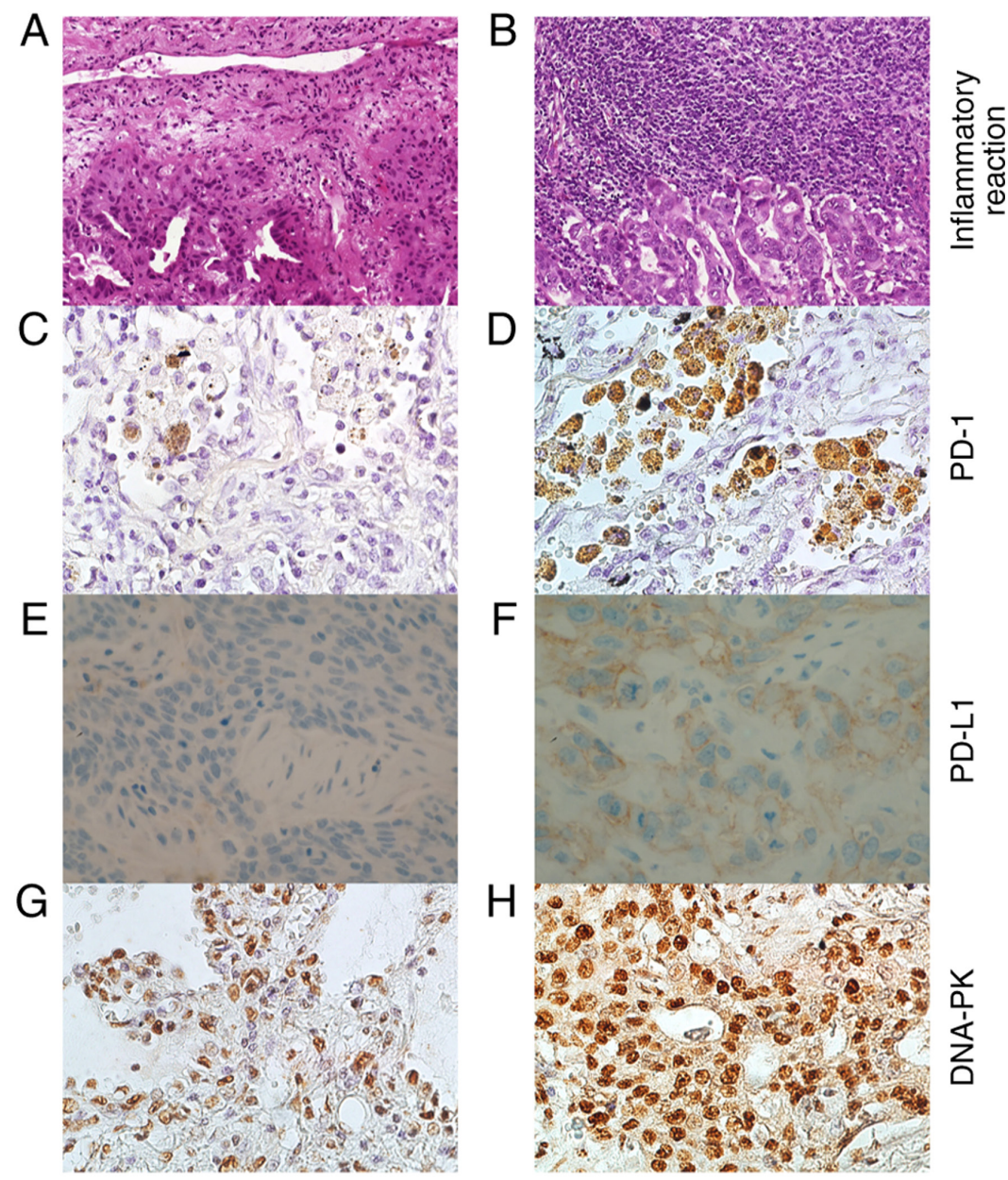

Figure 1. Representative inflammation and immunohistochemical expression of PD-1, PD-L1 and DNA-PK in NSCLC tissues. Representative images of NSCLC tissues with (A) trivial and (B) strong inflammatory reactions in tumor adjacent stroma (hematoxylin and eosin staining; magnification, $\mathrm{x} 200$ ). Immunohistochemical stains of NSCLC tissues with (C) scant PD-1 positive macrophages and (D) numerous PD-1 positive macrophages infiltrating the tumor (magnification, $\mathrm{x} 400$ ). Representative NSCLC sections with (E) scattered weak positively stained tumor cell nuclei for DNA-PK and (F) with diffuse and strong staining of cell nuclei for DNA-PK (magnification, $\mathrm{x} 400$ ). NSCLC samples with (G) negative and (H) positive PD-L1 expression (magnification, x400). PD-1, programmed cell death protein 1; PD-L1, programmed cell death ligand 1; DNA-PK, DNA-dependent protein kinase; NSCLC, non-small cell lung cancer.

According to IHC, the PD-1 expression originated from the lymphocytes present in the biopsy specimen.

Expression levels of PD-1, PD-L1 (22C3 pharmDx) and DNA-PK are depicted in Table II. In the evaluated parameters, we found significant differences in the subgroups for PD-1 and PD-L1. As shown in the Table II, there were more PD-1 positive cells in the tumor tissues collected from males than female patients $(\mathrm{P}=0.03)$. In addition, $\mathrm{PD}-1$ positive cells were low in number, when strong inflammation was present $(\mathrm{P}=0.03)$. Furthermore, we found that PD-L1 was strongly expressed in 'current smokers' patients than non-smokers $(\mathrm{P}=0.025)$. For DNA-PK expression, we did not find any notable differences in the selected clinical parameters (male vs. female, current vs. non-smoker), histological form (adenocarcinoma vs. squamous cell cancer) or the extent of inflammation.

Correlation analyses. The results of the correlation analysis are depicted in Fig. 2.

The analysis was based on patient's individual values and revealed a significant association between one of the targets of immune checkpoint inhibitors and tumor cell DNA-PK. Most importantly, we found a positive correlation between the number of PD-1 positive cells and tumor cell DNA-PK expression $(\mathrm{P}=0.027)$. The biopsies from three patients had extremely high levels of PD-1 expression; still, we found no physiologically relevant criteria to exclude these patients from the study.

We compared the results of two different PD-L1 immunohistochemistry assays before performing PD-L1 correlation analyses. This is because recent studies raised questions about analytical and clinical comparability of different PD-L1 assays used in clinical trials and practice as the assays utilize various staining platforms and may have their own scoring systems $(26,28,29)$. Indeed, we found that immunohistochemical stainings of the two PD-L1 antibodies used in our study did not correlate $(\mathrm{P}=0.651)$. Therefore, we used the results of clinically validated PD-L1 antibody (22C3 pharmDx) for PD-L1 and DNA-PK correlation analysis. In contrast to PD-1, no correlation between PD-L1 and DNA-PK was detected $(\mathrm{P}=0.926)$.

Correlation analysis of mRNA sequencing data available in the cancer genome atlas (TCGA) database. Finally, we explored whether the correlations established in this study could be derived based on mRNA or proteomic data available 
Table II. Expression of PD-1, PD-L1 (22C3 pharmDx) and DNA-PK in non-small cell lung cancer $(\mathrm{n}=121)$.

\begin{tabular}{|c|c|c|c|c|c|c|}
\hline \multirow[b]{2}{*}{ Variable } & \multicolumn{2}{|c|}{ PD-1 } & \multicolumn{2}{|c|}{ PD-L1 } & \multicolumn{2}{|c|}{ DNA-PK } \\
\hline & Mean \pm SEM & P-value & Mean \pm SEM & P-value & Mean \pm SEM & P-value \\
\hline Sex & & 0.030 & & 0.667 & & 0.733 \\
\hline Male & $17.6 \pm 6.0$ & & $18.5 \pm 3.3$ & & $86.1 \pm 1.0$ & \\
\hline Female & $5.9 \pm 2.0$ & & $13.5 \pm 4.2$ & & $86.7 \pm 1.6$ & \\
\hline Histology & & 0.127 & & 0.500 & & 0.157 \\
\hline Adenocarcinoma & $20.2 \pm 11.1$ & & $17.6 \pm 5.2$ & & $88.0 \pm 1.4$ & \\
\hline Squamous cell cancer & $11.6 \pm 4.2$ & & $18.4 \pm 3.6$ & & $85.4 \pm 1.2$ & \\
\hline Smoking status & & 0.259 & & 0.025 & & 0.814 \\
\hline Current smoker & $16.4 \pm 5.5$ & & $19.5 \pm 3.1$ & & $86.3 \pm 1.0$ & \\
\hline Non-smoker & $6.6 \pm 3.2$ & & $1.4 \pm 0.8$ & & $86.1 \pm 2.1$ & \\
\hline Inflammation score & & 0.030 & & 0.212 & & 0.149 \\
\hline No or weak inflammation (score $0-2$ ) & $18.2 \pm 6.2$ & & $19.0 \pm 3.4$ & & $86.3 \pm 1.0$ & \\
\hline Strong inflammation (score 3) & $5.0 \pm 1.5$ & & $13.9 \pm 4.8$ & & $85.8 \pm 1.6$ & \\
\hline
\end{tabular}

Data were analyzed using a Student's t-test. PD-1, programmed cell death protein 1; PD-L1, programmed cell death ligand 1; DNA-PK, DNA-dependent protein kinase.

in the public databases, The Cancer Genome Atlas (TCGA) and Human Protein Atlas (HPA).

As per TCGA database, a positive correlation was observed in mRNA levels of DNA-PK (PRKDC) and PD-L1 (CD274) (Spearman $\mathrm{P}=0.039$; Fig. S1) and a negative correlation was observed in mRNA levels of DNA-PK (PRKDC) and PD-1 (PDCD1) (Spearman $\mathrm{P}=0.0088$; Fig. S2). According to HPA, staining of all three proteins of interest (DNA-PK, PD-L1, and PD-1) can observed in lung cancer tissue samples, yet the patient-dependent variability is remarkably high for all cases.

\section{Discussion}

Blockade of inhibitory immune checkpoints PD-1/PD-L1 has become a valid treatment option for several tumors including advanced NSCLC $(7,8,30)$. Moreover, immunotherapy is increasingly used in combination with cytotoxic treatments, such as chemotherapy $(9,13)$. Although the combined treatments in NSCLC are more effective, the underling mechanisms that lead to higher antitumor activity are not fully understood. Therefore, the aim of our retrospective study was to describe the relationship between the expression of immune checkpoints (PD-1, PD-L1) and DNA-PK, which is a part of key pathway involved in repairing cytotoxic cancer therapy induced damage.

Till today, immunohistochemical expression level of PD-L1 on tumor cells is the most advanced biomarker in NSCLC. Several phase-III clinical studies have reported better treatment responses in the patients with higher tumor proportion score of PD-L1. The latter has been clearly shown in monotherapy studies in both, first- and second-line treatment of NSCLC patients $(7,8,30)$. Similarly, in the recently published study on lung adenocarcinoma patients, a positive relation was found between higher PD-L1 expression and longer overall survival wherein pembrolizumab plus pemetrexed and a platinum-based drug combination was used (9). Nevertheless, in patients with squamous cell carcinoma, the relationship between higher PD-L1 expression and longer overall survival was not detected (13). The latter indicates that the clinical usefulness of PD-L1 as a biomarker in patients receiving immunotherapy with cytotoxic agents may be less clear given that the combination treatments improved outcomes over chemotherapy across all categories of PD-L1 tumor proportion scores $(9,13)$. Therefore, the development of biomarkers for anti-PD-1/PD-L1 and chemotherapy combinations requires better understanding of PD-1 and PD-L1 expression profiles that is complemented with the markers, which are important in cytotoxic cancer therapy.

In our retrospective study, we first analyzed the expression of PD-1, PD-L1 and DNA-PK according to the clinical parameters (male vs. female, current vs. non-smoker), histological form (adenocarcinoma vs. squamous cell cancer) and the extent of inflammation. In all the evaluated parameters, we detected significant differences for PD-1 and PD-L1. Significantly higher number of positive PD-1 cells were seen in the tumor tissues from males, which supported the observations in the previous studies, wherein higher PD-1 scores were found in males (31). Indeed, it was shown that immunotherapy tends to be more effective in male cancer patients, when compared to female patients, whereas higher expression of PD-1 and PD-L1 was suggested as one of the reasons for the increased efficacy $(32,33)$.

Our study also revealed that NSCLC tissues with no or trivial visual inflammation had higher number of $\mathrm{PD}-1$ positive cells. It is well known that there are key histological differences between acute and chronic inflammation, especially in terms of leukocyte types that are predominantly present in the tissue (polymorphonuclear neutrophils vs. macrophages and lymphocytes) (34). Additionally, numerous studies showed that PD-1 expression can be found in multiple immune cell types, including $\mathrm{T}$ cells, B cells, macrophages as well as dendritic cells (35). In tumors, chronic inflammation leads to permanent stimulation of $\mathrm{T}$ cells, which promotes $\mathrm{T}$ cell exhaustion that 
A

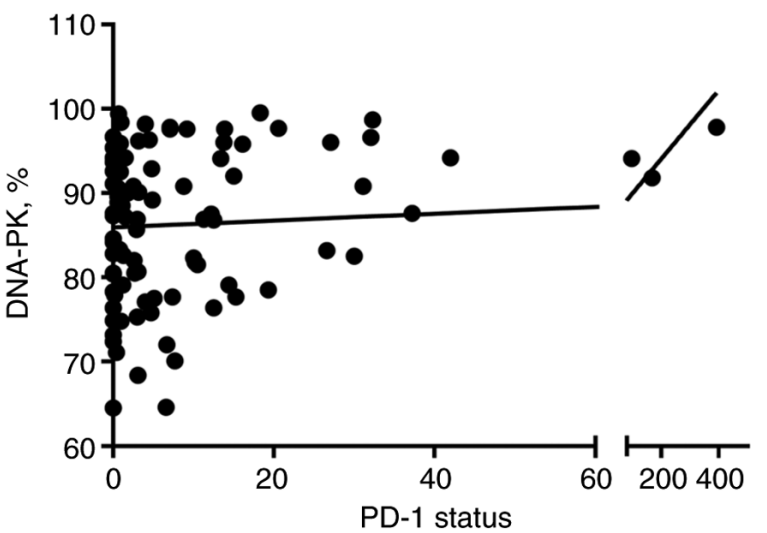

B

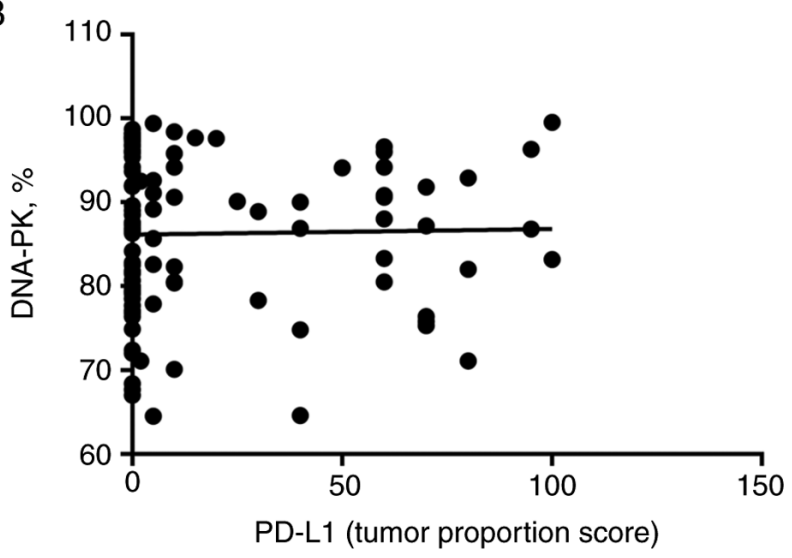

C

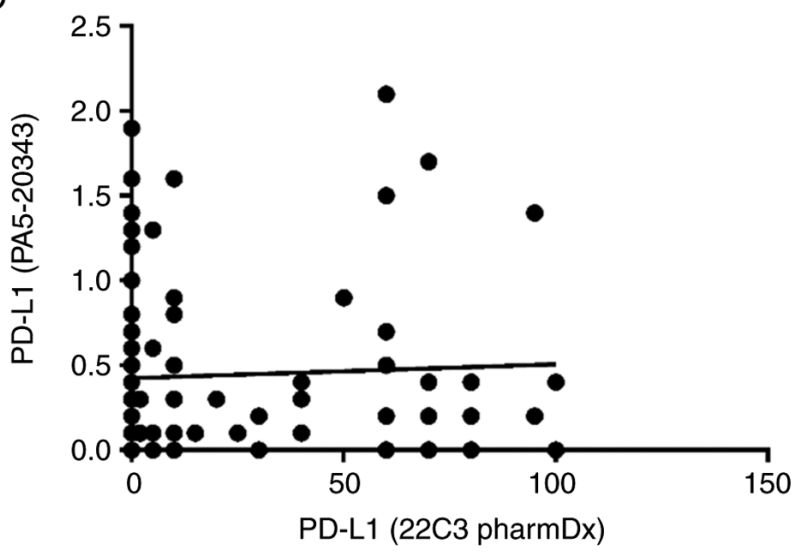

Figure 2. Results of nonparametric Spearman correlation analysis. (A) PD-1 (positive cells) and DNA-PK (proportion of positive tumor cells) correlation analysis $(\mathrm{P}=0.027)$. The $\mathrm{X}$-axis is segmented to demonstrate the outliers (with high expression of PD-1) as a separate group. (B) PD-L1 (tumor proportion score) and DNA-PK (proportion of positive tumor cells) correlation analysis ( $\mathrm{P}=0.926)$. (C) PD-L1 (22C3 pharmDx) and PD-L1 (PA5-20343) correlation analysis $(\mathrm{P}=0.651)$. $\mathrm{PD}-1$, programmed cell death protein $1 ; \mathrm{PD}-\mathrm{L} 1$, programmed cell death ligand 1; DNA-PK, DNA-dependent protein kinase.

finally results in upregulation of co-inhibitory receptors such as PD-1 (36). Therefore, it is reasonable to believe that higher number of PD-1 positive cells in the tumor's tissues with no or trivial inflammation as seen in our study was mainly due to inhibitory tumor microenvironment.

Recent studies have raised questions about analytical and clinical comparability of different PD-L1 assays used in clinical trials and practice because the assays utilize various IHC antibodies, staining platforms and may have their own scoring systems $(26,28,29)$. Here, we showed that the results of different IHC PD-L1 antibodies do not correlate. Therefore, it is very important to use clinically relevant antibodies even in retrospective studies.

PD-L1 immunohistochemistry with clinically validated antibody also revealed that the expression of PD-L1 was significantly higher in the NSCLC patients with 'current smokers' status. Several meta-analyses and other studies showed that high PD-L1 expression was positively correlated with different clinical and pathological features in lung cancer patients, such as male gender, smoking status, higher histological grade, larger tumor size, positive lymph nodal metastasis and TNM stage (37-39). Our study did not find any association between PD-L1 and other clinical and the selected pathological characteristics. It could be due to several reasons; first, our patient groups were not well balanced. Additionally, we were unable to rule out tumor heterogeneity, which was showed influential for PD-L1 expression in NSCLC as reported recently (40).

Although we did not detect significant differences in tumor cell DNA-PK expression in the selected clinical and pathological characteristics, the most intriguing finding of this study was, a significant positive correlation between PD-1/PD-L1 axis and DNA-PK expression based on individual patient values. The patients whose tumors had higher number of PD-1 positive cells, also showed higher proportion of DNA-PK positive tumor cells.

As mentioned earlier, DNA-PK, a serine/threonine protein kinase, consists of a catalytic subunit (DNA-PKcs) and Ku heterodimer that consists of Ku70 and Ku80 subunits (41). DNA-PK is involved in repairing DNA double-strand breaks (DSBs) via three main pathways: classical non-homologous end-joining NHEJ (C-NHEJ), alternative NHEJ (A-NHEJ) and homologous recombination (HR) (42). It has been widely accepted that the ability of cancer cells to repair therapeutically induced DNA damage impacts anti-cancer therapeutic efficacy to a major extent $(19,43,44)$. In our study, more than $80 \%$ of cancer cells (on average) were seen to express DNA-PK. Accordingly, in previously published studies, significantly higher DNA-PK expression was detected in NSCLC than adjacent normal tissues (45). Moreover, DNA-PK expression level serves as a biomarker for predicting a response to cytotoxic therapy and thereby survival, since worse treatment outcome was reported in the patients with high levels of DNA-PK $(45,46)$. Furthermore, the inhibition of DNA-PK was shown to enhance chemosensitivity as well as radiosensitivity in NSCLC $(24,25)$.

Interactions between DNA-PK and PD-1/PD-L1 axis are not fully explored. Previously, it was showed that nuclear $\gamma \mathrm{H} 2 \mathrm{AX}$ (unique histone subunit, which serves as a sensor of double-stranded DNA damage) expression was positively associated with PD-L1 expression in squamous cell lung carcinoma (47). Similarly, upregulation of PD-L1 expression in cancer cells was reported in response to DSBs (48). Moreover, as per TCGA database, a positive correlation was observed in mRNA levels of DNA-PK (PRKDC) and PD-L1 (CD274) (Fig. S1) (27). These evidences suggested that DNA damage response in tumor cells generates an immune response that further upregulates PD-L1 expression in tumor cells. Higher PD-L1 expression in the tumors cells consecutively sensitizes them to anti PD-L1 therapy. According to TCGA database, there is a negative correlation between the DNA-PK (PRKDC) and PD-1 (PDCD1) (Fig. S2), yet this does not 
directly contradict our findings: the biopsy samples used for IHC contain several types of cells, and hence protein staining can reveal correlations that are intrinsically different to those provided by mRNA sequencing in tumor cells only. This fact underlines the ultimate strength of our study.

Here, we showed that the patients, whose tumors had higher number of PD-1 positive cells, also had a higher proportion of DNA-PK positive tumor cells. Although the most well-known role of DNA-PK is, its involvement in DNA damage response pathways, this kinase also participates in other functions, e.g., promotion of genomic stability, regulation of hypoxic response via hypoxia inducible factor (HIF) dependent and HIF-independent mechanisms. In addition, DNA-PK affects metabolism, participates in transcriptional regulation of hormone receptors, and activates innate immune system $(41,42,49)$. Various studies have established the role of DNA-PK in transcriptional programs that operate biological processes, such as epithelial to mesenchymal transition (EMT), nuclear receptor signaling and inflammatory responses (22). However, importance of PD-1 and DNA-PK association in NSCLC is not known. Therefore, detailed studies are required regarding the mechanisms that are responsible for higher expression of PD-1 and DNA-PK in tumors and further their effect on the efficacy of combined treatments in NSCLC.

This report has several limitations, including retrospective nature of the study and absence of equally balanced study groups in terms of patient number. Furthermore, we used tumor tissue samples in our study. As opposed to NSCLC cell lines, tissue samples may contain not only cancerous cells but also other components native to the tumor microenvironment which may affect the results of the study. In spite of the limitations, the authors showed that, there is a significant positive correlation between PD-1/PD-L1 axis and DNA-PK expression in NSCLC. Clear understanding of underlying mechanisms is of high importance to develop predictive and prognostic molecular markers for the combination treatment of immune checkpoint inhibitors and cytotoxic cancer therapies. In our further studies, we will focus on the tumor side of the PD-1/PD-L1 signaling axis to investigate combined effects of immunotherapy and chemotherapy in a simplified system, represented by the PD-L1 positive vs. PD-L1 negative cell lines.

\section{Acknowledgements}

Not applicable.

\section{Funding}

The study was supported by the Estonian Society of Clinical Oncologists (grant no. A171063).

\section{Availability of data and materials}

The datasets used and/or analyzed during the current study are available from the corresponding author on reasonable request.

\section{Authors' contributions}

MS, JJ, MK and DL planned and guided the current study, analyzed the data and wrote the manuscript. JN, LM, HA, AV,
AM, MB, TV, MS and HT were responsible for histology, IHC and scoring. All authors read and approved final draft of the manuscript. MS and JJ confirm the authenticity of all the raw data.

\section{Ethics approval and consent to participate}

Research Ethics Committee of University of Tartu approved the study.

\section{Patient consent for publication}

Not applicable.

\section{Competing interests}

$\mathrm{JJ}$ is an advisory board member for AstraZeneca and MSD, and has received research funding from AstraZeneca. The other authors declare that they have no competing interests.

\section{References}

1. Didkowska J, Wojciechowska U, Manczuk M and Łobaszewski J: Lung cancer epidemiology: Contemporary and future challenges worldwide. Ann Transl Med 4: 150, 2016.

2. Siegel RL, Miller KD and Jemal A: Cancer statistics, 2015. CA Cancer J Clin 65: 5-29, 2015.

3. Planchard D, Popat S, Kerr K, Novello S, Smit EF, Faivre-Finn C, Mok TS, Reck M, Van Schil PE, Hellmann MD, et al: Metastatic non-small cell lung cancer: ESMO clinical practice guidelines for diagnosis, treatment and follow-up. Ann Oncol 29 (Suppl 4): iv192-iv237, 2018.

4. Yang JC, Wu YL, Schuler M, Sebastian M, Popat S, Yamamoto N, Zhou $\mathrm{C}$, Hu CP, O'Byrne K, Feng J, et al: Afatinib versus cisplatin-based chemotherapy for EGFR mutation-positive lung adenocarcinoma (LUX-Lung 3 and LUX-Lung 6): Analysis of overall survival data from two randomised, phase 3 trials. Lancet Oncol 16: 141-151, 2015.

5. Paz-Ares L, de Marinis F, Dediu M, Thomas M, Pujol JL, Bidoli P, Molinier O, Sahoo TP, Laack E, Reck M, et al: Maintenance therapy with pemetrexed plus best supportive care versus placebo plus best supportive care after induction therapy with pemetrexed plus cisplatin for advanced non-squamous non-small-cell lung cancer (PARAMOUNT): A double-blind, phase 3, randomised controlled trial. Lancet Oncol 13: 247-255, 2012.

6. Brahmer J, Reckamp KL, Baas P, Crinò L, Eberhardt WE, Poddubskaya E, Antonia S, Pluzanski A, Vokes EE, Holgado E, et al: Nivolumab versus docetaxel in advanced squamous-cell non-small-cell lung cancer. N Engl J Med 373: 123-135, 2015.

7. Borghaei H, Paz-Ares L, Horn L, Spigel DR, Steins M, Ready NE, Chow LQ, Vokes EE, Felip E, Holgado E, et al: Nivolumab versus docetaxel in advanced nonsquamous non-small-cell lung cancer. N Engl J Med 373: 1627-1639, 2015.

8. Reck M, Rodriguez-Abreu D, Robinson AG, Hui R, Csőszi T, Fülöp A, Gottfried M, Peled N, Tafreshi A, Cuffe S, et al: Pembrolizumab versus chemotherapy for PD-L1-positive non-small-cell lung cancer. N Engl J Med 375: 1823-1833, 2016.

9. Gandhi L, Rodriguez-Abreu D, Gadgeel S, Esteban E, Felip E, De Angelis F, Domine M,Clingan P,Hochmair MJ,Powell SF, et al: Pembrolizumab plus chemotherapy in metastatic non-small-cell lung cancer. N Engl J Med 378: 2078-2092, 2018.

10. Attili I, Passaro A, Pavan A, Conte P, De Marinis F and Bonanno L: Combination immunotherapy strategies in advanced non-small cell lung cancer (NSCLC): Does biological rationale meet clinical needs? Crit Rev Oncol Hematol 119: 30-39, 2017.

11. Langer CJ: Emerging immunotherapies in the treatment of non-small cell lung cancer (NSCLC): The role of immune checkpoint inhibitors. Am J Clin Oncol 38: 422-430, 2015.

12. Rocco D, Della Gravara L, Battiloro C and Gridelli C: The role of combination chemo-immunotherapy in advanced non-small cell lung cancer. Expert Rev Anticancer Ther 19: 561-568, 2019. 
13. Paz-Ares L, Luft A, Vicente D, Tafreshi A, Gümüs M, Mazières J, Hermes B, Çay Şenler F, Csőszi T, Fülöp A, et al: Pembrolizumab plus chemotherapy for squamous non-small-cell lung cancer. N Engl J Med 379: 2040-2051, 2018.

14. Yu W, Hua Y, Qiu H, Hao J, Zou K, Li Z, Hu S, Guo P, Chen M, Sui S, et al: PD-L1 promotes tumor growth and progression by activating WIP and $\beta$-catenin signaling pathways and predicts poor prognosis in lung cancer. Cell Death Dis 11: 506, 2020.

15. Rizvi NA, Hellmann MD, Snyder A, Kvistborg P, Makarov V, Havel JJ, Lee W, Yuan J, Wong P, Ho TS, et al: Cancer immunology. Mutational landscape determines sensitivity to PD-1 blockade in non-small cell lung cancer. Science 348: 124-128, 2015.

16. Mouw KW, Goldberg MS, Konstantinopoulos PA and D'Andrea AD: DNA damage and repair biomarkers of immunotherapy response. Cancer Discov 7: 675-693, 2017.

17. Ricciuti B, Recondo G, Spurr LF, Li YY, Lamberti G, Venkatraman D, Umeton R, Cherniack AD, Nishino M, Sholl LM, et al: Impact of DNA damage response and repair (DDR) gene mutations on efficacy of PD-(L)1 immune checkpoint inhibition in non-small cell lung cancer. Clin Cancer Res 26: 4135-4142, 2020

18. Lamberti G, Andrini E, Sisi M, Federico AD and Ricciuti B Targeting DNA damage response and repair genes to enhance anticancer immunotherapy: Rationale and clinical implication. Future Oncol 16: 1751-1766, 2020

19. Gavande NS, VanderVere-Carozza PS, Hinshaw HD, Jalal SI, Sears CR, Pawelczak KS and Turchi JJ: DNA repair targeted therapy: The past or future of cancer treatment? Pharmacol Ther 160: 65-83, 2016.

20. Blackford AN and Jackson SP: ATM, ATR, and DNA-PK: The trinity at the heart of the DNA damage response. Mol Cell 66 : 801-817, 2017.

21. Jette $\mathrm{N}$ and Lees-Miller SP: The DNA-dependent protein kinase: A multifunctional protein kinase with roles in DNA double strand break repair and mitosis. Prog Biophys Mol Biol 117: 194-205, 2015

22. Medová M, Medo M, Hovhannisyan L, Muñoz-Maldonado C, Aebersold DM and Zimmer Y: DNA-PK in human malignant disorders: Mechanisms and implications for pharmacological interventions. Pharmacol Ther 215: 107617, 2020.

23. Sak A, Stuschke M, Wurm R, Schroeder G, Sinn B, Wolf G and Budach V: Selective inactivation of DNA-dependent protein kinase with antisense oligodeoxynucleotides: Consequences for the rejoining of radiation-induced DNA double-strand breaks and radiosensitivity of human cancer cell lines. Cancer Res 62 : 6621-6624, 2002.

24. Yanai M, Makino H, Ping B, Takeda K, Tanaka N, Sakamoto T, Yamaguchi K, Kodani M, Yamasaki A, Igishi T and Shimizu E: DNA-PK inhibition by NU7441 enhances chemosensitivity to topoisomerase inhibitor in non-small cell lung carcinoma cells by blocking DNA damage repair. Yonago Acta Med 60: 9-15, 2017.

25. Azad A, Jackson S, Cullinane C, Natoli A, Neilsen PM, Callen DF, Maira SM, Hackl W, McArthur GA and Solomon B: Inhibition of DNA-dependent protein kinase induces accelerated senescence in irradiated human cancer cells. Mol Cancer Res 9: 1696-1707, 2011

26. Hirsch FR, McElhinny A, Stanforth D, Ranger-Moore J, Jansson M, Kulangara K, Richardson W, Towne P, Hanks D, Vennapusa B, et al: PD-L1 immunohistochemistry assays for lung cancer: Results from phase 1 of the blueprint PD-L1 IHC assay comparison project. J Thorac Oncol 12: 208-222, 2017.

27. The Cancer Genome Atlas Program-National Cancer Institute. https://www.cancer.gov/about-nci/organization/ccg/research/structural-genomics/tcga. Accessed February 20, 2021

28. Tsao MS, Kerr KM, Kockx M, Beasley MB, Borczuk AC, Botling J, Bubendorf L, Chirieac L, Chen G, Chou TY, et al: PD-L1 immunohistochemistry comparability study in real-life clinical samples: Results of blueprint phase 2 project. J Thor Oncol 13: 1302-1311, 2018

29. Kintsler S, Cassataro MA, Drosch M, Holenya P, Knuechel R and Braunschweig T: Expression of programmed death ligand (PD-L1) in different tumors. Comparison of several current available antibody clones and antibody profiling. Ann Diagn Pathol 41: 24-37, 2019.
30. Rittmeyer A, Barlesi F, Waterkamp D, Park K, Ciardiello F, von Pawel J, Gadgeel SM, Hida T, Kowalski DM, Dols MC, et al: Atezolizumab versus docetaxel in patients with previously treated non-small-cell lung cancer (OAK): A phase 3, open-label, multicentre randomised controlled trial. Lancet 389: 255-265, 2017.

31. D'Incecco A, Andreozzi M, Ludovini V, Rossi E, Capodanno A, Landi L, Tibaldi C, Minuti G, Salvini J, Coppi E, et al: PD-1 and PD-L1 expression in molecularly selected non-small-cell lung cancer patients. Br J Cancer 112: 95-102, 2015

32. Conforti F, Pala L, Bagnardi V, De Pas T, Martinetti M, Viale G, Gelber RD and Goldhirsch A: Cancer immunotherapy efficacy and patients' sex: A systematic review and meta-analysis. Lancet Oncol 19: 737-746, 2018.

33. Wang S, Cowley LA and Liu XS: Sex differences in cancer immunotherapy efficacy, biomarkers, and therapeutic strategy. Molecules 24: 3214, 2019.

34. Oort J and Scheper RJ: Histopathology of acute and chronic inflammation. Agents Actions Suppl: 25-30, 1977.

35. Qin W, Hu L, Zhang X, Jiang S, Li J, Zhang Z and Wang X: The diverse function of PD-1/PD-L pathway beyond cancer. Front Immunol 10: 2298, 2019.

36. Jubel JM, Barbati ZR, Burger C, Wirtz DC and Schildberg FA: The role of PD-1 in acute and chronic infection. Front Immunol 11: 487, 2020.

37. Zhang M, Li G, Wang Y, Wang Y, Zhao S, Haihong P, Zhao H and Wang Y: PD-L1 expression in lung cancer and its correlation with driver mutations: A meta-analysis. Sci Rep 7: 10255, 2017.

38. Calles A, Liao X, Sholl LM, Rodig SJ, Freeman GJ, Butaney M, Lydon C, Dahlberg SE, Hodi FS, Oxnard GR, et al: Expression of PD-1 and its ligands, PD-L1 and PD-L2, in smokers and never smokers with KRAS-mutant lung cancer. J Thor Oncol 10: 1726-1735, 2015 .

39. Pawelczyk K, Piotrowska A, Ciesielska U, Jablonska K, Gletzel-Plucinska N, Grzegrzolka J, Podhorska-Okolow M, Dziegiel P and Nowinska K: Role of PD-L1 expression in non-small cell lung cancer and their prognostic significance according to clinicopathological factors and diagnostic markers. Int J Mol Sci 20: 824, 2019.

40. McLaughlin J, Han G, Schalper KA, Carvajal-Hausdorf D, Pelekanou V, Rehman J, Velcheti V, Herbst R, LoRusso P and Rimm DL: Quantitative assessment of the heterogeneity of PD-L1 expression in non-small-cell lung cancer. JAMA Oncol 2: 46-54, 2016.

41. Mohiuddin IS and Kang MH: DNA-PK as an emerging therapeutic target in cancer. Front Oncol 9: 635, 2019.

42. Goodwin JF and Knudsen KE: Beyond DNA repair: DNA-PK function in cancer. Cancer Discov 4: 1126-1139, 2014.

43. Li L, Zhu T, Gao YF, Zheng W, Wang CJ, Xiao L, Huang MS, Yin JY, Zhou HH and Liu ZQ: Targeting DNA damage response in the radio(Chemo)therapy of non-small cell lung cancer. Int J Mol Sci 17: 839, 2016

44. Kase M, Vardja M, Lipping A, Asser T and Jaal J: Impact of PARP-1 and DNA-PK expression on survival in patients with glioblastoma multiforme. Radiother Oncol 101: 127-131, 2011.

45. Xing J, Wu X, Vaporciyan AA, Spitz MR and Gu J: Prognostic significance of ataxia-telangiectasia mutated, DNA-dependent protein kinase catalytic subunit, and $\mathrm{Ku}$ heterodimeric regulatory complex $86-\mathrm{kD}$ subunit expression in patients with nonsmall cell lung cancer. Cancer 112: 2756-2764, 2008.

46. Hu S, Qu Y, Xu X, Xu Q, Geng J and Xu J: Nuclear survivin and its relationship to DNA damage repair genes in non-small cell lung cancer investigated using tissue array. PLoS One 8: e74161, 2013.

47. Osoegawa A, Hiraishi H, Hashimoto T, Takumi Y, Abe M, Takeuchi H, Miyawaki M, Okamoto T and Sugio K: The positive relationship between $\gamma \mathrm{H} 2 \mathrm{AX}$ and PD-L1 expression in lung squamous cell carcinoma. In Vivo 32: 171-177, 2018.

48. Sato H, Niimi A, Yasuhara T, Permata TBM, Hagiwara Y, Isono M, Nuryadi E, Sekine R, Oike T, Kakoti S, et al: DNA double-strand break repair pathway regulates PD-L1 expression in cancer cells. Nat Commun 8: 1751, 2017.

49. Damia G: Targeting DNA-PK in cancer. Mutat Res 821: 111692 , 2020.

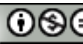

This work is licensed under a Creative Commons Attribution-NonCommercial-NoDerivatives 4.0 International (CC BY-NC-ND 4.0) License. 